\title{
Optofluidic Microring Dye Laser
}

\author{
Zhenyu Li, Zhaoyu Zhang, Axel Scherer, and Demetri Psaltis, Fellow, IEEE \\ Department of Electrical Engineering, California Institute of Technology, \\ Pasadena, CA 91125 USA \\ zhenyu@caltech.edu
}

\begin{abstract}
We demonstrated, for the first time, an optofluidic microring dye laser on a monolithic poly(dimethylsiloxane) (PDMS) chip. Laser threshold of $9.2 \mathrm{~nJ}$ was obtained with a single mode liquid-core waveguide based microring cavity.
\end{abstract}

\section{INTRODUCTION}

$\mathrm{T}$ HE combination of high quality (Q)-factor and small size has attracted applications of microring resonators in numerous applications such as lasers, filters, modulators and sensors [1]. In particular, ultralow threshold lasers have been made using microring-like cavities [1]. In the context of 'lab-on-a-chip' applications, microring structures may enable the implementation of low threshold liquid dye lasers on a microfluidic chip which can be pumped by a laser diode or a high power LED to make fully integrated devices. Recently, we demonstrated a single mode distributed feedback (DFB) liquid dye laser on a monolithic PDMS chip [2] using a soft lithographic replica molding method. Here we show the same fabrication process and material system can be used to make microring liquid dye lasers which can be monolithically integrated with PDMS microfluidics and the required pump threshold power is within the reach of commercial laser diodes. This is, to our knowledge, the first integrated optical microring liquid dye laser ever demonstrated.

\section{PRINCIPLES OF OPERATION}

As shown in Fig. 1, the optofluidic microring dye laser was fabricated on a monolithic PDMS chip using replica molding soft lithography as described in Ref. [2][3]. A microfluidic channel when filled with liquid of higher refractive index than that of PDMS (1.406, GE RTV 615) acts as a channel optical waveguide. When the cross section dimensions of the channel are 2 um by $3 \mathrm{um}$ and the index contrast is less than 0.003 , the waveguide supports only the two fundamental spatial modes. A 300 um diameter microring resonator which is side coupled to a bus waveguide forms the laser cavity. For a single layer device, a fused coupling region (as shown in Fig. 2) is used to enable the transportation of dye solution into the microring. This introduces additional scattering losses which lower the loaded $\mathrm{Q}[4]$ and the coupling coefficient is expected to be large. These problems can be solved using a double layer structure in which

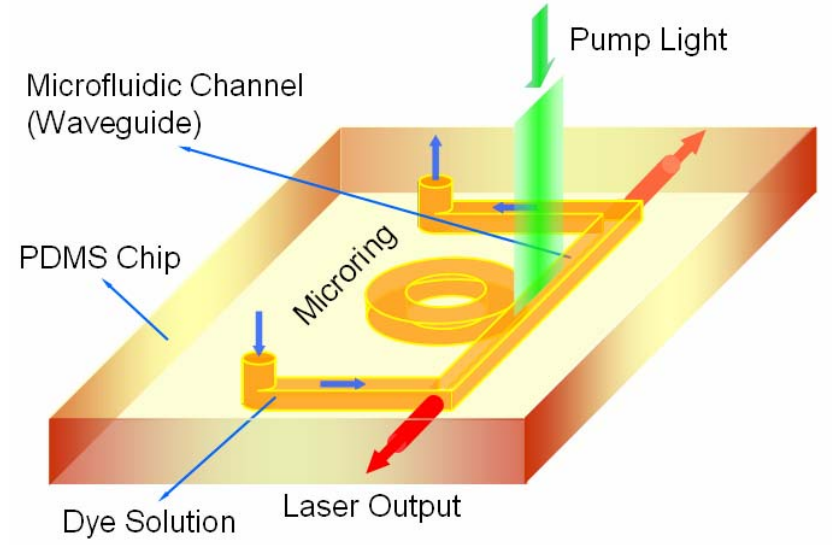

Fig.1. Schematic diagram of an optofluidic microring dye laser chip.

the microring resonator and the bus waveguide are located in separate layers and vertical coupling scheme can be used. The gain medium is a $1 \mathrm{mM}$ solution of Rhodamine $6 \mathrm{G}$ in a methanol and ethylene glycol mixture with refractive index of 1.409. The pump light are 532nm Q-switched Nd:YAG laser pulses with 6 nanosecond pulse width. The pump light is focused by a cylindrical lens to a 500 um by $1000 \mathrm{um}$ area covering the microring and a portion of the bus waveguide. Laser light coupled out of the microring into the bus waveguide can get further amplified by the pumped dye solution in the waveguide.

The resonant wavelengths of a microring laser are determined by the condition

$$
m \lambda_{m}=2 \pi n_{e f f} R
$$

where $\lambda_{m}$ is the $m$ th resonant wavelength, $n_{e f f}$ is the effective index of the guided mode and $\mathrm{R}$ is the ring radius. For a $300 \mathrm{um}$ diameter ring, this gives a free spectral range (FSR) of $0.25 \mathrm{~nm}$ around $580 \mathrm{~nm}$. It's clear from this expression that the optofluidic integration enables tunable laser wavelength by changing $n_{\text {efff }}, \mathrm{R}$ or $m$. The effective index can be varied by changing the refractive index of the liquid core. Due to the very low Young's modulus of PDMS ( 750kPa [5]), the ring radius and shape can be mechanically changed by simply stretching or compressing the chip. Finally, the resonance order can be chosen by using different dye molecules which cover different spectral regions. 


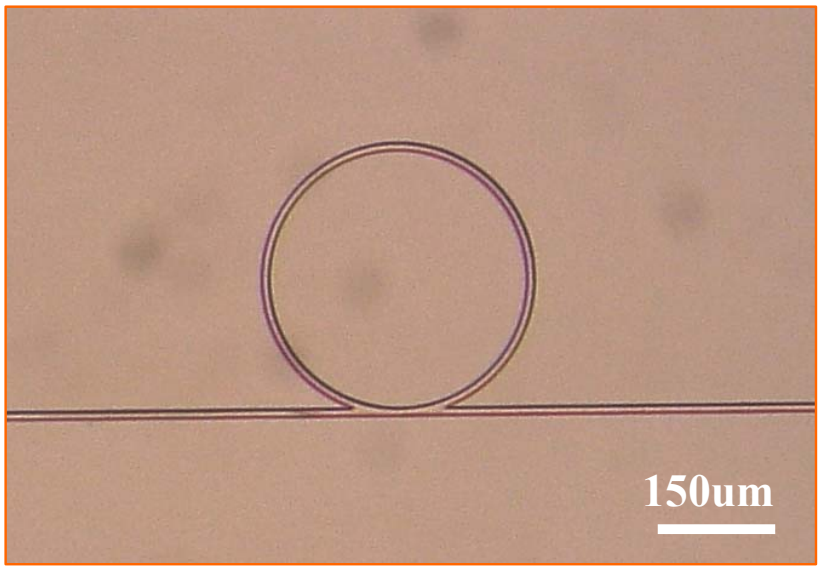

(a)

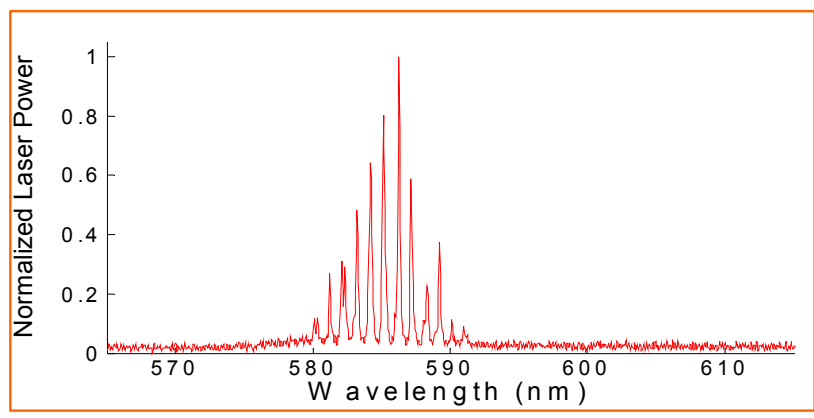

(b)

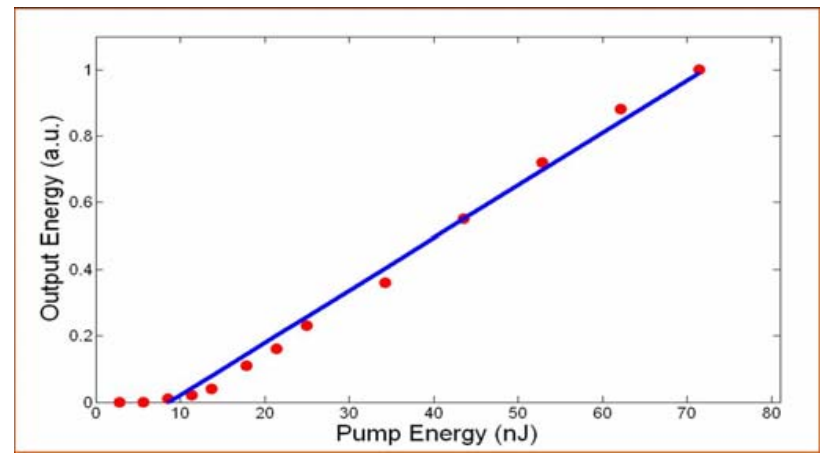

(c)

Fig.2. (a) Optical micrograph of an optofluidic microring resonator in PDMS. (b) A typical measured lasing spectrum of an optofluidic microring dye laser with a 300um diameter ring. (c) L-L curve. The laser threshold is $9.2 \mathrm{~nJ}$.

\section{RESULTS AND DISCUSSION}

The transparent PDMS chip was placed on a high reflectivity mirror so that the transmitted pump light can be reflected back to the laser cavity. This not only makes more efficient use of the pump light but also lowers the laser threshold. Furthermore, this makes possible a vertical resonant structure at the pump wavelength which can further reduce the laser threshold. The dye solution was pressure driven into the microfluidic channel by applying 10 psi pressure at the inlet port. A $10 \mathrm{X}$ microscope objective was used to collect the emission light from one edge of the chip and deliver it to a spectrometer with $0.1 \mathrm{~nm}$ resolution (Ocean Optics HR4000). A typical multiple longitudinal mode lasing spectrum is shown in Fig. 2 (b) where the linewidth of each mode is $<0.1 \mathrm{~nm}$. The measured FSR agrees with the predicted value $0.25 \mathrm{~nm}$. A plot of laser output energy versus the absorbed pump energy is shown in Fig. 2 (c) The threshold pump energy is only $9.2 \mathrm{~nJ}$ due to the small size of the laser. This is well within the reach of the output power of commercially available laser diodes.

This device can be used an active biosensor without any modification, in which the analytes can be either functionally attached to the channel wall or directly injected into the liquid flow and the shift in lasing wavelength is detected.

In the future, we plan to study the mechanical and fluidic tunability of this laser. In addition, in order to build a single mode microring dye laser, we'll use the Vernier effect in which two microring resonators with slightly different diameters will be used as the frequency selective element. Another interesting direction is to achieve mode locking by introducing saturable absorbers.

\section{CONCLUSION}

We have demonstrated, for the first time, a microfabricated optofluidic microring dye laser on a monolithic PDMS chip. The fabrication and operation of the laser chip is fully compatible with PDMS based microfluidics technology [6]. Besides low threshold laser applications, such microring resonators can be used to build optical filters, biosensors, ultrashort pulse generators on a chip.

\section{ACKNOWLEDGMENT}

Z. Y. Li thanks Dr. Lan Yang for helpful discussions.

\section{REFERENCES}

[1] K. J. Vahala, "Optical microcavities," Nature, vol. 424, no. 6950, pp. 839-846, August (2003).

[2] Z. Y. Li, Z. Zhang, T. Emery, A. Scherer, and D. Psaltis, "Single mode optofluidic distributed feedback dye laser," Opt. Express, vol. 14, pp. 696-701 (2006). http://www.opticsinfobase.org/abstract.cfm?URI=oe-14-2-696

[3] Y. N. Xia and G. M. Whitesides, "Soft lithography," Annu. Rev. Mater. Sci., vol. 28, pp. 153-184 (1998).

[4] B. E. Little, J. S. Foresi, G. Steinmeyer, E. R. Thoen, S. T. Chu, H. A. Haus, E. P. Ippen, L. C. Kimerling, and W. Greene, "Ultra-compact $\mathrm{Si}_{-} \mathrm{SiO}_{2}$ microring resonator optical channel dropping filters, ” IEEE, Photon. Tech. Lett., vol. 10, no. 4, pp 549-551, April (1998).

[5] J. C. McDonald and G. M. Whitesides, "Poly(dimethylsiloxane) as a material for fabricating microfluidic devices," Acc. Chem. Res., vol. 35, pp. 491-499 (2002)

[6] M. A. Unger, H. P. Chou, T. Thorsen, A. Scherer, S. R. Quake, "Monolithic microfabricated valves and pumps by multilayer soft lithography," Science, vol. 288, pp. 113-116 (2000). 